\title{
NHLBI Coronary Artery Dissection Type B
}

National Cancer Institute

\section{Source}

National Cancer Institute. NHLBI Coronary Artery Dissection Type B. NCI Thesaurus. Code C119589.

Parallel tracts or double lumen separated by a radiolucent area during contrast injection with no persistence after dye clearance. (Adapted from: Coronary artery ang iographic changes after PTCA: Manual of Operations NHLBI PT CA Registry 1985-6:9) 